\title{
Lane-changing and freeway capacity: a Bayesian inference stochastic model
}

\author{
Marcel Sala \& Francesc Soriguera* \\ BIT - Barcelona Innovative Transportation, Civil and Environmental Engineering \\ Department, UPC-BarcelonaTech, Barcelona, Spain.
}

\begin{abstract}
This paper presents a new stochastic computational model for determining freeway capacity reduction as a result of lane-changing activity. The probability density function for the maximum flow that can be sustained on a freeway for a given lane-changing activity level is obtained. The obtained results can be used to support freeway management strategies aiming to mitigate the negative consequences of lane-changing in freeway capacity. A pilot test using empirical data obtained from the B-23 freeway accessing the city of Barcelona proves the validity of the modelling approach.
\end{abstract}

\section{INTRODUCTION}

Freeway congestion is a recurrent phenomenon on freeways around the world. When demand exceeds capacity, congestion arises. This cause-effect relationship is not deterministic, as freeway capacity is affected by multiple random factors. Capacity is defined as the maximum sustained flow that can be supported by an infrastructure, and should be seen as a stochastic variable. Supporting this stochastic approach, several works have found that traffic breakdown can happen at the same freeway section for significantly different flow levels (Elefteriadou et al., 1995; Lorenz \& Elefteriadou, 2000; Minderhoud et al., 1997; Muñoz \& Daganzo, 2002; Okamura et al., 2000; Persaud et al., 1998; Yeon, et al., 2009). In this context, stochastic capacity estimation methods have received increased attention (Geistefeldt \& Brilon, 2009; Ozbay \& Ozguven, 2007; Polus \& Pollatschek, 2001) and stochasticity has been incorporated into traffic management tools (Jia et al., 2011). Many factors may affect freeway capacity. For instance, adverse weather conditions have a negative impact and relationships between a reduced capacity and rainfall intensity have been established (Brilon et al., 2005; Highway Capacity Manual, 2010; Ibrahim \& Hall, 1994; Lamm et al., 1987; Smith et al., 2004). Freeway capacity also depends on the prevailing drivers' typology, being larger when drivers are mainly commuters instead of occasional drivers (Highway Capacity Manual, 2010). Although less studied, lane-changing intensity is another factor contributing to the variability of freeway capacity (Cassidy et al., 2010; Menendez \& Daganzo, 2007).

Beyond stochasticity, it has also been proven that when traffic breaks down, freeway capacity is reduced. This harmful phenomenon, known as "capacity drop", has been repeatedly observed around the world (Banks, 1991; Cassidy \& Rudjanakanoknad, 2005; Cassidy \& Bertini, 1999; Chung et al., 2007; Hall \& Agyemang-Duah, 1991; Oh \& Yeo, 2012; Patire \& Cassidy, 2011; Srivastava \& Geroliminis, 2013; Yuan et al., 2015). The typical capacity drop in active freeway bottlenecks ranges from $3 \%$ to $18 \%(\mathrm{Oh} \& \mathrm{Yeo}$, 2012). While the existence of the capacity drop has been extensively demonstrated, the traffic mechanism behind it is still under debate. In the literature, two traffic characteristics closely related to capacity reductions have been explored: $i$ ) lane-changing, and ii) vehicles' sluggish acceleration when leaving a queue.

On the one hand, lane-changing activity has been found to cause capacity reductions near bottlenecks in different scenarios. In Cassidy and Rudjanakanoknad (2005) systematic lane-changing from the shoulder to faster lanes was found to cause traffic breakdown. This usually happens near ramp merge junctions, where the capacity drop is dependent on the lane-changing induced by large clusters of vehicles entering the freeway (Elefteriadou et al., 2005). Also, Patire and Cassidy (2011) observed a significant flow reduction after a lane-changing increase due to speed variations between lanes. The massive lane-changing happening at speed drops generates traffic disturbances, which end up in generalized queueing on all lanes (Hatakenaka et al., 2004). In contrast, when a HOV lane is activated on a congested freeway, it smoothens traffic, even in the adjacent general-purpose lanes, reducing the amount of lane-changing and increasing the freeway throughput (Cassidy et al., 2010; Menendez \& Daganzo, 2007). 
On the other hand, vehicles' sluggish acceleration has also been found to be related to the capacity drop (Chen et al., 2014; Goñi-Ros et al., 2016; Knoop et al, 2009; Laval \& Daganzo, 2006; Leclercq et al., 2011; Yuan et al., 2019). Vehicles changing lanes near bottlenecks are observed to create larger gaps in the arriving lane due to their limited acceleration when moving from a slow to a faster lane. This leads to a total throughput reduction. These findings are in accordance with Yuan et al. (2015), where it is reported that the slower the speed in the queue, the larger the capacity drop.

The negative effects of lane-changing are not limited to being a main capacity drop contributor. Also, they trigger traffic oscillations (Mauch \& Cassidy, 2002; Wang \& Coifman, 2008) and stop and go waves (Ahn \& Cassidy, 2007; Oh and Yeo, 2015). Moreover, lane changes are found to globally increase delay (Coifman et al., 2006), as the time saved in the exiting lane is smaller than the induced delay in the arriving lane.

In spite of all these very laudable observations and findings, the lack of adequate and reliable empirical databases has been a recurrent problem in order to increase our knowledge on lane-changing behavior and its effects. This situation implies that few modeling approaches are found in the literature, mainly due to the difficulties in their validation. In one of the first attempts (Laval and Daganzo, 2006) proposed a model with few parameters in order to allow calibration with few data; still, authors acknowledged that it remained to be validated when empirical data was available. Other researchers performed ad-hoc experiments to validate their lane-changing models. Take as an example (Sun \& Kondyli, 2010; Sun \& Elefteriadou, 2012) where an instrumented vehiclebased experiment was designed to analyze urban lanechanging scenarios. Alternatively, (Marczak et al., 2014) conducted a descriptive empirical analysis of a trajectory data set from a freeway weaving section and constructed a lane-changing database. This data has been used to validate several models attempting to reproduce the macroscopic lane-changing effects on freeway capacity (Marczak et al., 2015; Chen \& Ahn, 2018).

In light of this data scarcity, big efforts have been made to construct reliable databases to support the research community. Take as an example the NGSIM project (Federal Highway Administration, 2006, 2015). Unfortunately, the freeway traffic trajectories database resulting from the project, suffers from large errors in the lateral vehicle position (Punzo, et al., 2011). Thus, directly estimating lane-changing from NGSIM database implies big errors. In order to correct this issue, Montanino and Punzo (2015) did a meticulous data filtering job to improve lane assignment in the NGSIM dataset. Still, some errors remain as Coifman and $\mathrm{Li}$ (2017) point out. This later work highlights some sources of the errors, like vehicles (mostly motorcycles) traveling on the hard shoulder, or in between lanes. Still, the NGSIM database has been used to validate either macroscopic (Jin, 2010) and microscopic lane-changing models (Jin et al., 2019).

In conclusion, to date, the relationship between freeway capacity and lane-changing activity has only been quantified for a handful of different flows and particular freeway configurations, precluding researchers to develop and calibrate stochastic lane-changing models. The lack of an analytical model, yielding quantitative knowledge, has also prevented traffic flow optimization in terms of lanechanging.

One possibility to overcome this limitation is to use Bayesian networks or Markov chains to simulate additional data. Examples of this type of solution can be found in different research fields, including civil engineering and transportation operations research (Kosgodagan et al., 2017; Yuen \& Huang, 2018; Huang \& Beck, 2018; Ghofrani et al., 2019). The applications in safety related research are especially relevant, because fatal accidents are fortunately a rare occurrence and thus scarce in the datasets. Take as examples the works of (Castillo et al., 2017a; Castillo et al., 2017b; de Oña et al., 2011; Deublein et al., 2015; Hossain \& Muromachi, 2012) in the field of road safety, and (Castillo et al., 2016a; Castillo et al., 2016b) in that of railway safety. Bayesian networks are also used to forecast traffic flows (Mihaylova et al., 2007; Sun et al., 2006; Yin et al., 2002; Lv et al., 2015). In spite of the recent increased interest on these data driven techniques, to the authors' best knowledge this is the first work using them to analyze the stochastic relationship between freeway capacity and lane-changing.

In the present paper, a Bayesian inference computational approach is proposed to obtain a stochastic model relating the lane-changing normalized ratio (i.e. the expected number of lane-changes of one vehicle in one $\mathrm{km}$ of travel) and the maximum observed freeway flow. The model needs to be calibrated with empirical data, and a pilot test with data from the B-23 freeway, accessing the city of Barcelona (Soriguera \& Sala, 2014; Soriguera et al., 2017) is presented in the paper. The proposed modelling approach should not be interpreted as a causal model to estimate the number of lanechanges given some explicative factors, but a model which establishes the effects of lane-changing activity (whatever their reasons are) on freeway capacity. The modeling results quantify to what extend lane-changing needs to be restricted in order to achieve larger capacities at freeways. Having better knowledge on capacity dependencies could be used for improved traffic management strategies (Hashemi \& 
Abdelghany, 2018; Tajalli \& Hajbabaie, 2018), and more accurate short-term traffic predictions (Liu, et al., 2018; Yao, et al., 2017). For instance, such strategies could be applied at critical links of the freeway network, for instance at bridges or tunnels, which are typical bottlenecks whose physical expansion is extremely expensive. Capacity could be increased by applying dynamic lane-changing limitations, because typically there is no need for mandatory lane changing in these freeway stretches. These findings represent advances to the current knowledge and specifically in the state-of-theart in the application of computational methods to traffic flow modeling.

The reminder of the paper is organized as follows. Because, the modeling approach is data driven, a descriptive analysis of lane-changing behavior is needed first, in order to postulate a candidate analytical model. This is done in Sections 2 and 3. Section 2 presents the traffic database, including a description of the methods used to process and aggregate the data. Next, Section 3 presents a descriptive analysis of the database, unveiling the relationship between the peaking of lane-changing and congestion shockwaves. Section 4 actually deals with the computational modeling, addressing the stochastic relation between lane-changing rates and the average flow per lane. The proposed model yields the probability density function for capacity. Finally, Section 5 outlines and highlights some of the conclusions obtained from the analysis.

\section{THE EMPIRICAL DATABASE}

This section contains a description of the database used in order to calibrate and validate the proposed stochastic model. Data was collected on the B-23 freeway accessing the city of Barcelona from the south-west (Figure 1). Measurements took place on the last $13 \mathrm{Km}$ stretch of the freeway in the inbound direction towards the city, during 7 different days (namely Day\#1 to Day\#7). In order to ensure similar demands and traffic conditions, data was only collected with good weather (clear skies) on Tuesdays, Wednesdays and Thursdays during June, 2013. See (Soriguera \& Sala, 2014) for a complete description of the data collection process and access to the database.

Figure 1 shows the lane-changing video surveillance zones (red interlane lines) and the traffic detectors close to them (grey vertical lines). Traditional traffic data (e.g. flow, average speed and detector occupancy as a proxy for traffic density) are obtained from detectors, either using traditional double loops or the newer non-intrusive devices. In any case, they are point detectors, in the sense that their measurements are taken on a freeway section of less than $10 \mathrm{~m}$. long. Thus, the spatial coverage of the measurements is limited, and spatial variables can only be indirectly derived from the measurements of consecutive detectors. This surveillance scheme is not suitable for measuring lane changes, which need to be observed over space and time. To that end, video camera surveillance is used. Detection zones were set over the camera coverage along the line dividing the lanes. Details about the retrieval of lane-changing data from video recordings, the different techniques used and their errors are provided in (Sala et al., 2019). Note that motorbike lane changes were discarded, as they travel quite often in-between lanes, especially in congestion, and generally represent a source of errors in lanechanging data (Coifman \& Li, 2017). In spite of this, note that motorbikes may impact the lane-changing behavior of surrounding vehicles. The analysis of such impacts would require additional video surveillance and data treatment, and it is left as an issue for further research. Finally, because some detection zones include, or are in the proximity of, on/off-ramps and weaving areas (e.g. detection zone 2309), two types of lane-changes may happen: i) mandatory lanechanges in order to change route, and ii) discretionary lanechanges in order to better accommodate drivers' preferences. Unfortunately, the available database does not discriminate lane-changes by their motivation.

\subsection{Configuration of the detection zones}

Each lane-changing detection zone has its distinct layout with respect to its neighboring traffic detectors. We can distinguish between three groups of configurations: I) detection zones in between traffic detectors. II) zones with only one detector at its downstream end; III) zones with one detector within the detection zone, without any other nearby detector, neither up- nor downstream. Therefore, there are camera detection zones with additional data from 2 detectors (Type I) and others with data from just one detector (Types II and III). Figure 2 defines the configuration of these types of detection zones. The parameters for each detection zone in the present case study are shown in Table 1.

\subsection{Available data and aggregation procedures}

Traffic variables measured by punctual detectors (i.e. flow, occupancy and average speed) were available per lane and for periods of 60 seconds. In turn, lane-changing maneuvers were available for each pair of adjacent lanes and without any spatial or temporal aggregation (i.e. individual maneuvers with their precise time and location were recorded). If needed, these data can be aggregated by section (i.e. all lanes), space, and/or time. 


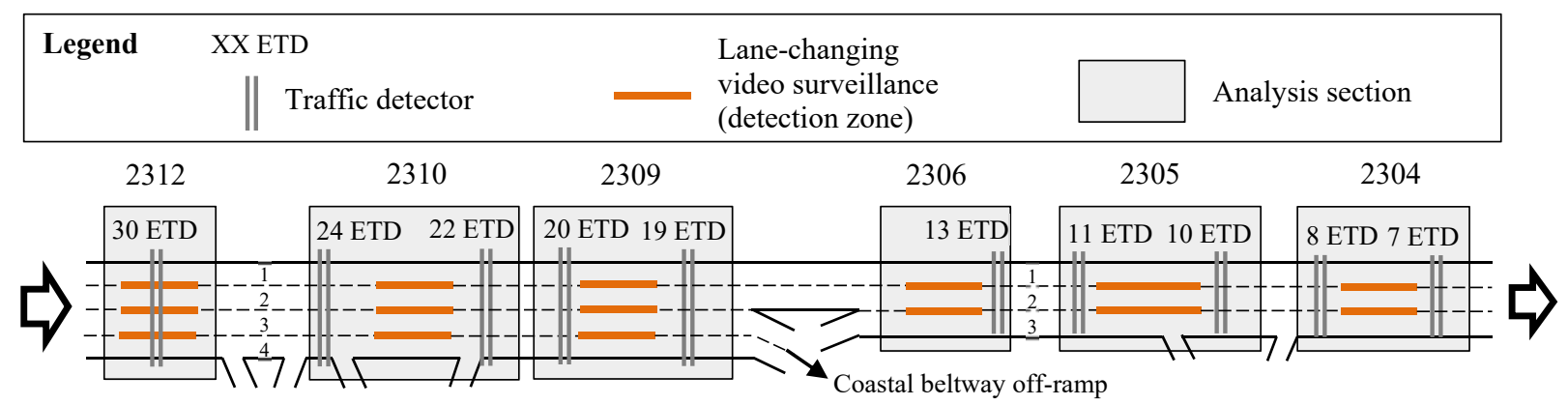

Figure 1. B-23 test site schematic layout and lane numbering. Note: Traffic direction goes from left to right; On- / Offramp representations mean that there is at least one of this type of junctions in between detectors.

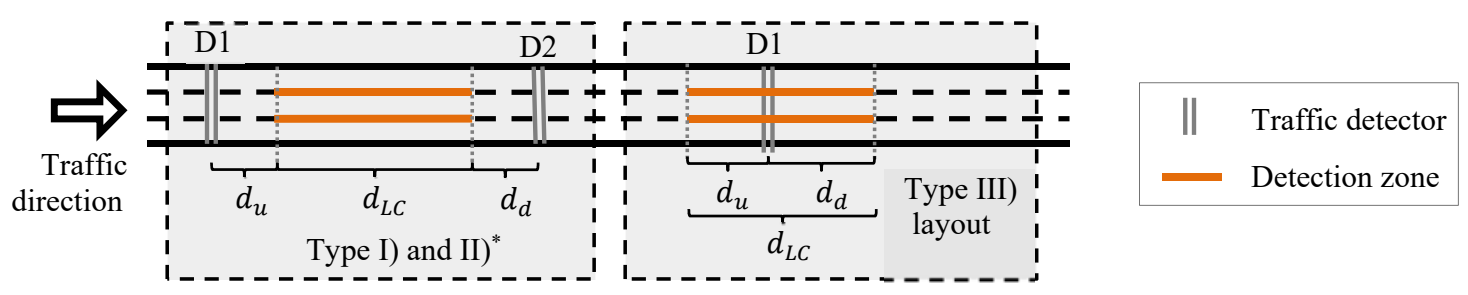

Figure 2. Different detection zone layouts. *In Type II detection zones, only one of the detectors (D1 or D2) is available.

Table 1

Configuration of the B-23 freeway detection zones.

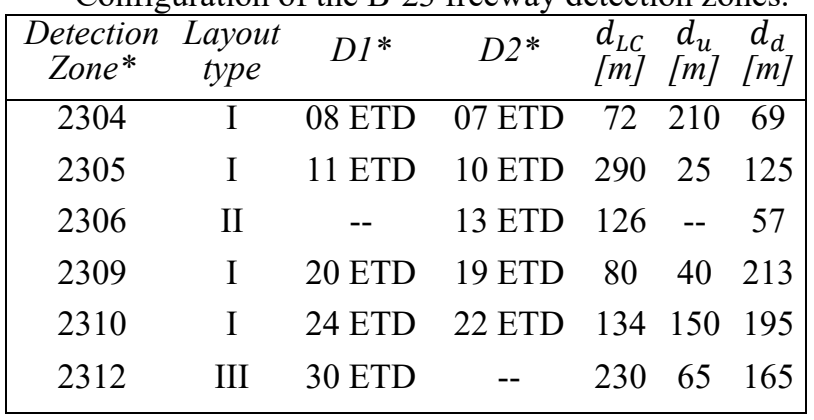

*Refers to the alias used in the traffic management center to identify the detector (see Figure 1).

Some caution is necessary when dealing with the less familiar lane-changing data. $n$ is defined as the sectional aggregated number of lane-changing maneuvers on a particular detection zone and during a particular observation period. Then, $n$ is influenced by: $i)$ the distance encompassed by the detection zone, $d_{L C}$; ii) the duration of the observation period, $\Delta t$; and iii) the average sectional traffic flow during the same period, $q_{(\Delta t)}$. This reflects the fact that, for a given freeway stretch under stationary traffic conditions, the longer the detection zone and the observation period, the more lane-changes are expected to be observed. In addition, the higher the traffic flow (i.e. veh/unit time), the larger the number of candidates to change lanes.

In order to normalize the lane-changing count $(n)$, two aggregated variables are proposed. First, the lane changing flow $(s)$ [maneuvers/unit time-unit space], defined as in Equation 1.

$$
s=\frac{n}{d_{L C} \cdot \Delta t}
$$

(s) expresses the number of measured lane-changing maneuvers $(n)$, extrapolated to one $\mathrm{km}$ of freeway during an observation period of one hour. This allows comparing the measurements at different test sites (i.e. with different lengths and different durations of observation).

Second, in order to take into account the effect of different circulating flows, the lane-changing normalized ratio $(r)$ [unit space ${ }^{-1}$ ] is defined as:

$$
r=\frac{s}{q_{(\Delta t)}}
$$

$(r)$ is simply a normalized version of $(s)$ considering the average traveling flow during the observation period, $q_{(\Delta t)}$. $(r)$ defines the expected number of lane-changes of one vehicle in one $\mathrm{km}$ of travel. Note that for Type I detection zones, $q_{(\Delta t)}$ is computed using the arithmetic mean of the vehicle counts at the two limiting detectors. 


\section{DESCRIPTIVE ANALYSIS: CONGESTION, SHOCKWAVES AND LANE-CHANGING}

The proposed descriptive analysis consists in the adequate processing and plotting of the previous data. This allows unveiling several interesting relationships between traffic states and lane-changing activity. Data processing includes the construction and plot of oblique cumulative curves. These curves were introduced by Cassidy and Windover (1995) and allow observing with richer detail many traffic features. Oblique cumulative curves, $\hat{X}(t)$, are obtained by plotting the cumulative sum of the sectional aggregation of the variable under analysis, $X(t)$, but subtracting a background value, $b_{0}$, close to the average magnitude of the variable during the period (see Equation 3).

$$
\hat{X}(t)=X(t)-b_{0} t
$$

The logic behind oblique cumulative curves is that by eliminating the large cumulative average, the plot magnifies the rate of change of the variable instead of showing the fairly constant increase of the cumulative sum. Oblique curves of flow, $\widehat{N}(t)$, occupancy, $\widehat{T}(t)$, and lane changing, $\hat{L}(t)$, are used (see Figures 3, 4 and 5), because they are especially suited to detect congestion, and allow unveiling the particular behavior of lanechanging in each period. In the following subsections, the detailed insights obtained from the temporal, spatial and corridor wide empirical analyses are presented.

\subsection{Lane-changing peaks in congested periods}

From the analysis of the database, it is found that lane changing recurrently peaks in congested periods. In addition, this phenomenon is particularly intense when transitioning between free flow and congested regimes. The previous assertions are supported by the evidences presented in Figure 3. For the sake of briefness, only few examples are presented, but the same behavior has been observed in every single congestion episode.

Figure 3 consists of three cumulative oblique curves, $\widehat{N}(t), \widehat{T}(t)$ and $\hat{L}(t) . \widehat{N}(t)$ is the cumulative count curve (i.e. the total number of vehicles that have crossed the section since the beginning of observation). $\widehat{T}(t)$ represents the same concept but considering the occupancy (i.e. the total cumulative time all vehicles spent on the detector since the beginning of observation). Finally, $\hat{L}(t)$ represents again the same concept but considering lane-changing maneuvers for the entire detection zone, including all lane pairs. Congestion is detected from these plots as an increase of the slope of the

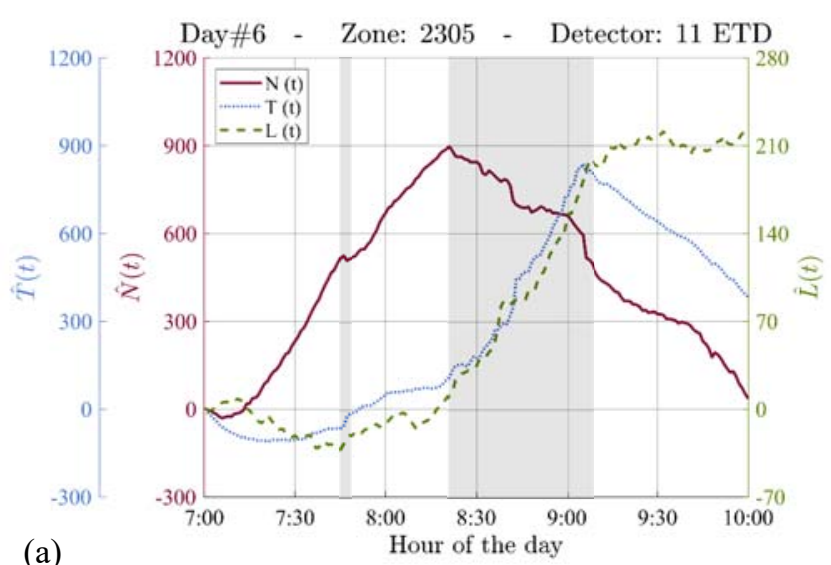

(a)

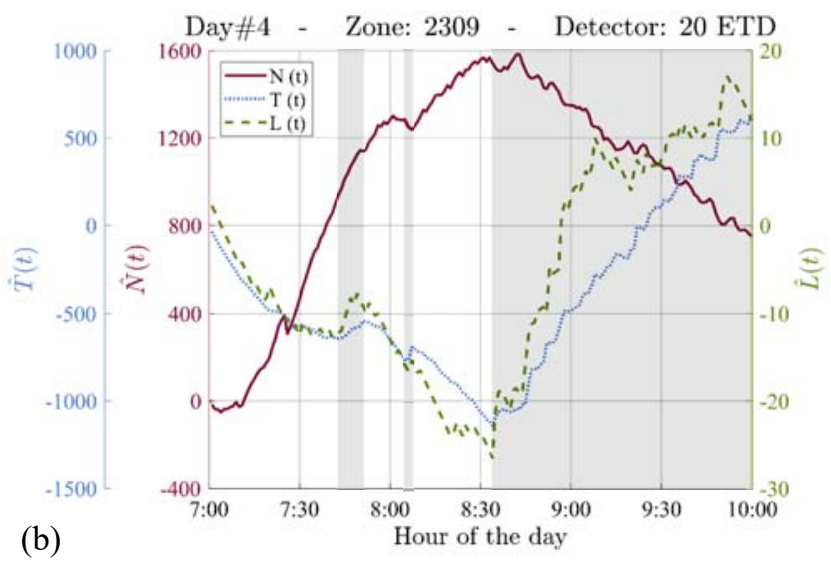

(b)

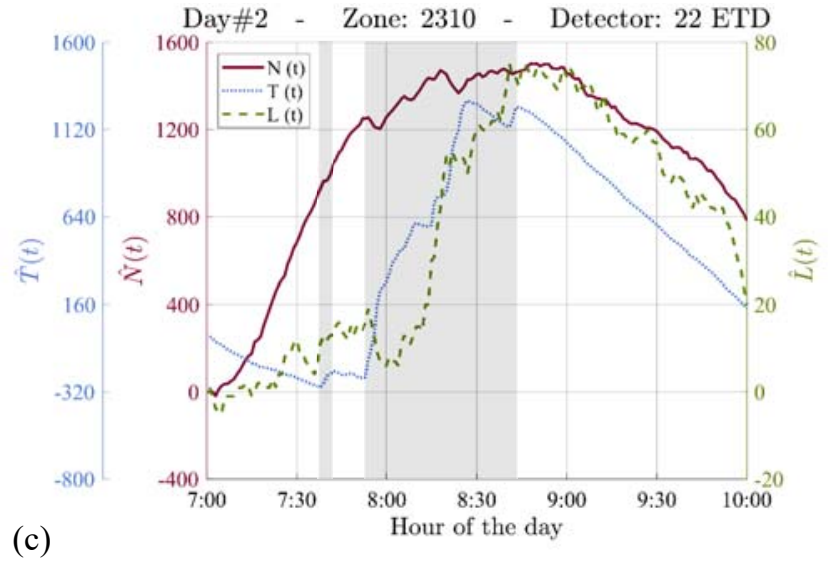

Figure 3. Flow, $\widehat{N}(t)$, occupancy, $\widehat{T}(t)$, and lanechanging, $\hat{L}(t)$, oblique cumulative plots in congested periods. (a), (b) and (c) correspond to different days and detection zones. Note: 1) Congested periods are shaded in light grey; 2) Background subtraction rates, $b_{0}$, for $\widehat{N}(t)$, $\widehat{T}(t)$ and $\widehat{L}(t)$ are respectively: a) $4700[\mathrm{veh} / \mathrm{h}], 1800[\mathrm{~s} / \mathrm{h}]$, $380[\mathrm{veh} / \mathrm{h}]$; b) $5000[\mathrm{veh} / \mathrm{h}], 3000[\mathrm{~s} / \mathrm{h}], 45[\mathrm{veh} / \mathrm{h}]$; c) 4600 [veh/h], $1900[\mathrm{~s} / \mathrm{h}], 240[\mathrm{veh} / \mathrm{h}]$. 
T-curve without an equivalent increase in the slope of the $\mathrm{N}$-curve (i.e. an increase of occupancy not corresponding with a simultaneous increase in flow). These periods are identified by visual inspection of the curves, as proposed in Cassidy and Windover (1995). In order to ease observations in Figure 3, congestion is shaded in light grey. Clearly, lane-changing rates increase in congestion, as shown by the increase of the slope of the $\hat{L}(t)$ during these periods.

\subsection{Lane-changing peaks in traffic regime transitions}

It is also found that the highest peaking of lane-changing rates happens during traffic regime transitions (i.e. from congestion to free-flowing or vice versa). Figure 4 shows the same previous oblique cumulative curves (as in Figure 3 ) for detection zone 2305. Figure 4a considers traffic data from the downstream detector (i.e. 10 ETD). Three peaks in the lane-changing rate (i.e. slope of the $\widehat{L}(t)$ curve) are observed (i.e. around 8:00; between 8:20 and 9:10; and between 9:40 and 9:50 approximately). In contrast, data from detector 10 ETD exhibits free-flowing conditions during the whole observation period (see Figure 4a).

The issue in detection zone 2305 is that while the downstream detector location (i.e. 10 ETD) is not congested, actually there is congestion within detection zone. A bottleneck exists, caused by the off-ramp located within the zone (see Figure 1). This off-ramp ends at a roundabout, which during peak periods reaches capacity and queues appear. These queues spill-back into the freeway mainline, spreading into the rightmost lanes and eventually congesting the entire freeway trunk. This can be observed in Figure 4b, equivalent to Figure $4 \mathrm{a}$ but considering data from the upstream detector (i.e. 11 ETD). It can be seen that congestion reaches detector 11 ETD three times, which approximately match the three surges in the lane-changing rates. The spatial analysis of lane-changing reveals that most maneuvers are located at the traffic regime transitions (i.e. at traffic shockwaves, around the back of the queue and around the bottleneck - head of the queue).
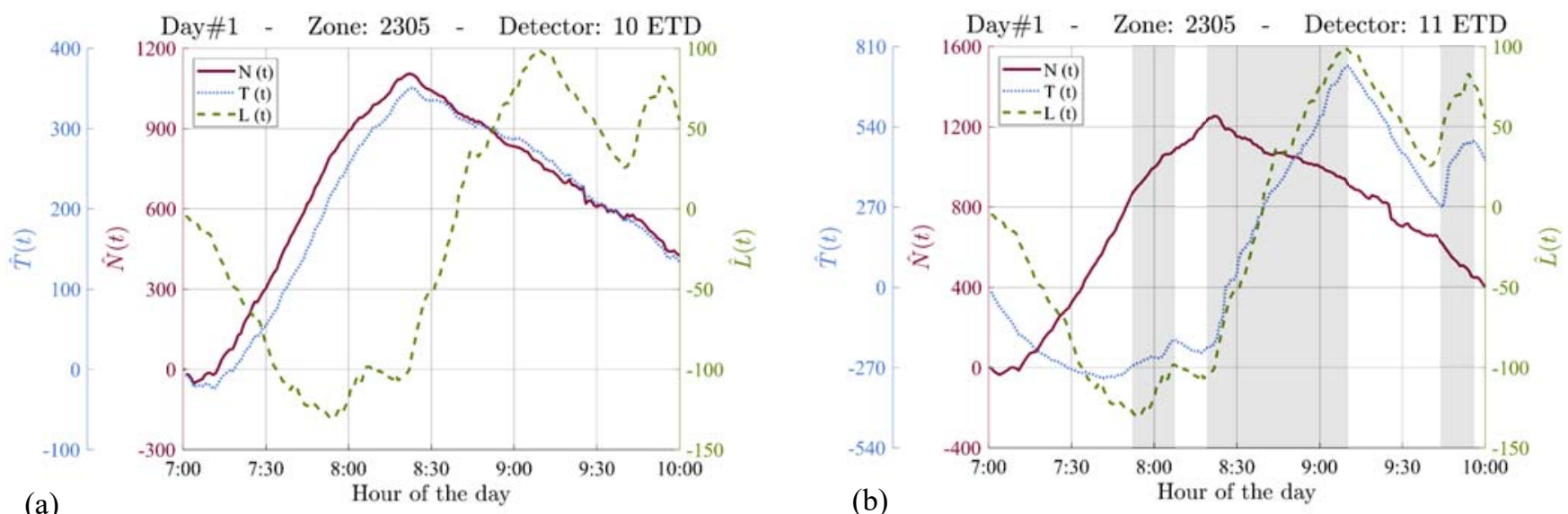

Figure 4. Effects of the bottleneck within detection zone 2305. Traffic data source: (a) Downstream detector 10 ETD; (b) Upstream detector 11 ETD. Note: 1) Congested periods are shaded in light grey; 2) Background subtraction rates, $b_{0}$, for $\widehat{N}(t)$, $\widehat{T}(t)$ and $\widehat{L}(t)$ are respectively: a) $3500[\mathrm{veh} / \mathrm{h}], 950[\mathrm{~s} / \mathrm{h}], 380[\mathrm{veh} / \mathrm{h}] ; \mathrm{b}) 4500[\mathrm{veh} / \mathrm{h}], 2000[\mathrm{~s} / \mathrm{h}], 380[\mathrm{veh} / \mathrm{h}]$.

Further evidence is provided in Figure 5, again equivalent to Figures 3 and 4 but for detection zone 2304. In Figure 5, lane-changing peaks between 9:10-9:20. This is precisely when a congestion dissolve shock wave travels from the upstream to the downstream detector in the section. This can be seen because at 9:10 the upstream section starts free flowing (Figure 5b), while the dissolving shockwave travelling in the direction of traffic reaches the downstream detector shortly after, at 9:20 (Figure 5a). 

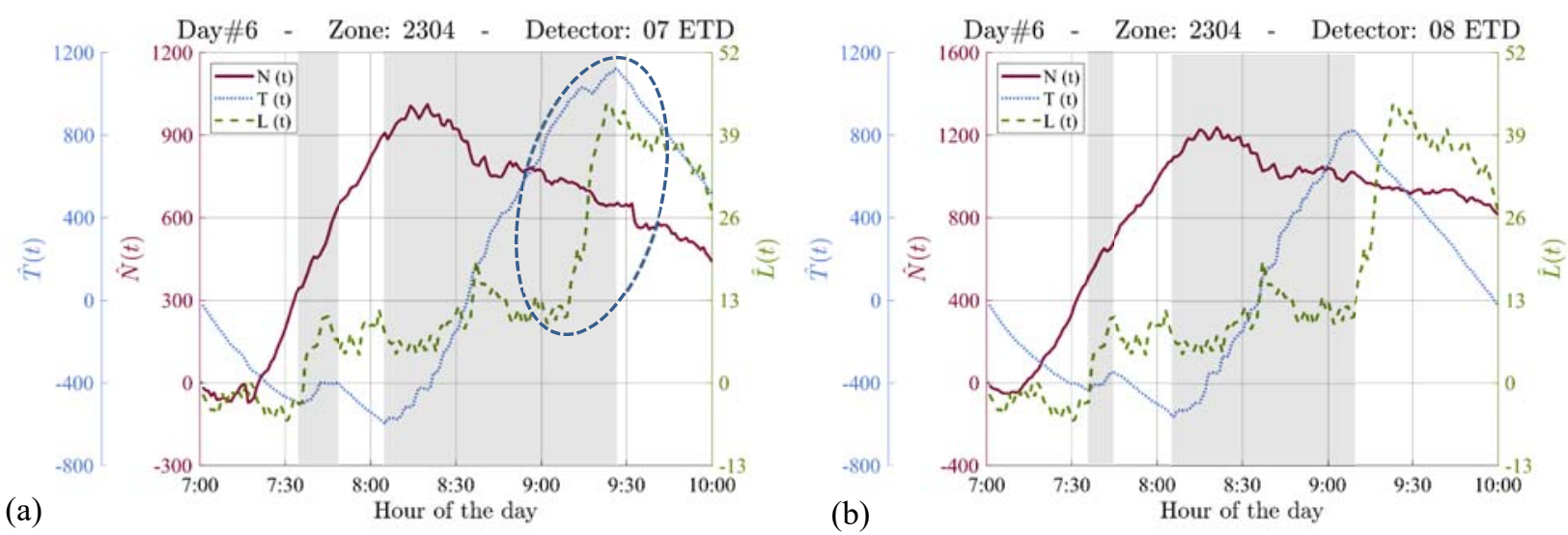

Figure 5. Flow, occupancy and lane-changing oblique cumulative plots during traffic regime transitions. (a) Downstream detector 07 ETD. (b) Upstream detector 08 ETD. Note: 1) Congested periods are shaded in light grey; 2) Background subtraction rates, $b_{0}$, for $\widehat{N}(t), \widehat{T}(t)$ and $\widehat{L}(t)$ are respectively: 4000 [veh/h], 2000 [s/h], 110 [veh/h] for both parts a) and b).

\section{THE STOCHASTIC RELATIONSHIP BETWEEN LANE-CHANGING AND FREEWAY CAPACITY}

From the descriptive analysis in the previous sections, it was observed that, as the average flow per lane $(\bar{q})$ increases, the maximum lane-changing normalized ratio $(r)$ decreases. In other words, for a given $r$, there exists a maximum flow that can be sustained, and this flow decreases with the increase in $r$. In the present section, it is postulated that this relationship, like many others involving freeway capacity, is stochastic in nature. Furthermore, data suggest that the relationship is heteroscedastic, specifically meaning that a larger variance of $r$ is observed for smaller average flows.

\subsection{The stochastic model}

The stochastic relationship between $r$ and the maximum observed flows, can be modeled by an analytical function where some of the parameters follow a probabilistic distribution. The proposed stochastic model describes $r$ as a heteroscedastic normal distribution where the mean and standard deviation decay polynomially with the average flow per lane, $\bar{q}$. This is:

$$
r[i] \sim \mathcal{N}\left(\mu[i], \sigma^{2}[i]\right)
$$

where $i=1 \div N$ refers to sectional observations of $\bar{q}[i]$ and $r[i]$, and Equations 5 and 6 define, respectively, the polynomial decay of the mean $(\mu)$ and standard deviation $(\sigma)$ of the normal distribution of $r$.

$$
\mu[i]=\alpha \cdot|Q-\bar{q}[i]|^{\gamma} \quad \alpha, \gamma \geq 0 ; \quad Q \geq \bar{q} \geq 0
$$

$$
\sigma[i]=\beta \cdot|Q-\bar{q}[i]|^{\delta} \quad \beta, \delta \geq 0 ; \quad Q \geq \bar{q} \geq 0
$$

According to the modeling assumptions, $\mu$ and $\sigma$ are modeled considering three random hyperparameters: $\alpha, \beta$ and $Q . \alpha$ and $\beta$ represent the decay rate of $\mu$ and $\sigma$, respectively, while $Q$ is the theoretical maximum per lane capacity in the absence of lane-changing. Note that $Q$ is generally not observable given the current conditions on freeways, and could only be observed by enforcing $r=0$. In turn, $\gamma$ and $\delta$ represent two deterministic calibration parameters defining the polynomial specification of the model. Figure 6 shows the graphical representation of this stochastic model and its dependencies.

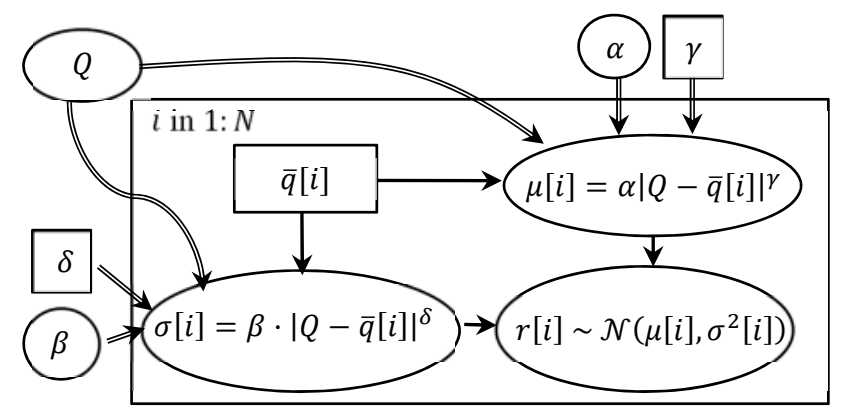

Figure 6. Graphical model of the relationship between the lane-changing normalized ratio, $r$, and the average flow per lane, $\bar{q}$.

Bayesian inference is applied in order to derive the posterior probability distribution of $r$ for a given average flow per lane, $\bar{q}$. The posterior distribution can then be used 
to estimate the parameters of the model and to predict the distribution and simulate new data points. In the Bayesian approach two antecedents are needed: a prior probability distribution and a likelihood function derived from a statistical model for the empirical data. Then, the posterior distribution can be solved via Bayes theorem:

$$
\begin{aligned}
& p(\mu, \sigma \mid \boldsymbol{R}, \alpha, \beta, Q, \gamma, \delta)= \\
& =\frac{p(\boldsymbol{R} \mid \mu, \sigma, \alpha, \beta, Q, \gamma, \delta) p(\mu, \sigma \mid \alpha, \beta, Q, \gamma, \delta)}{p(\boldsymbol{R} \mid \alpha, \beta, Q, \gamma, \delta)} \propto \\
& \propto p(\boldsymbol{R} \mid \mu, \sigma, \alpha, \beta, Q, \gamma, \delta) p(\mu, \sigma \mid \alpha, \beta, Q, \gamma, \delta)
\end{aligned}
$$

where:

- $\quad \boldsymbol{R}$ is the sample, a set of data points (i.e. $r[i], \bar{q}[i]$ for $i=1 \div N)$

- $p(\mu, \sigma \mid \boldsymbol{R}, \alpha, \beta, Q, \gamma, \delta)$ is the sought posterior distribution of the parameters defining the distribution of $r$ after considering the observed data.

- $\quad p(\boldsymbol{R} \mid \mu, \sigma, \alpha, \beta, Q, \gamma, \delta)$ is the likelihood (or sampling distribution); the distribution of the observed data conditional on their parameters.

- $p(\mu, \sigma \mid \alpha, \beta, Q, \gamma, \delta)$ is the prior distribution, the distribution of parameters before any data is observed.

- $\quad p(\boldsymbol{R} \mid \alpha, \beta, Q, \gamma, \delta)$ is the marginal likelihood (or model evidence), a normalization factor which does not affect the relative probabilities in the estimation of the model.

The prior distribution is obtained from Equations 5 and 6 , and assuming distributions for $\alpha, \beta$ and $Q$. These are chosen to be non-informative (i.e. very wide distributions) so that this does not affect the model calibration process, being the empirical data what drives the results. A Gamma $(0.001,0.001)$ distribution is chosen for $\alpha$ and $\beta$, because it is positive-definite and suitable for the small values expected for these parameters. For $Q$, a Normal $(2300,1000)$ is selected. This selection responds to the fact that the distribution should be centered around the a-priori capacity value. This is set to 2300 veh/h/lane following the HCM (Highway Capacity Manual, 2010) recommendation for a freeway with a free flow speed of $60 \mathrm{mph}(\sim 100 \mathrm{Km} / \mathrm{h})$, which is approximately observed in most of the B-23 freeway. The probabilities of negative values are negligible in this case. In turn, the values for $\gamma$ and $\delta$ are calibrated through a sensitivity analysis selecting the polynomial specification that best fits the available data (see Section 4.3)

To predict the distribution of a new, unobserved data point, $\tilde{R}=(\tilde{r}, \tilde{\bar{q}})$, the posterior predictive distribution is used. This can be computed as the distribution of the new data point, marginalized over the posterior. This is:

$$
\begin{gathered}
p(\tilde{R} \mid \boldsymbol{R}, \alpha, \beta, Q, \gamma, \delta)= \\
=\int p(\tilde{R} \mid \mu, \sigma) p(\mu, \sigma \mid \boldsymbol{R}, \alpha, \beta, Q, \gamma, \delta)
\end{gathered}
$$

However, a generally simpler alternative to computing the integral in Equation 8 is to use sampling algorithms based on Monte Carlo Markov Chain techniques (MCMC). These algorithms rely on the fact that it is possible to compute any statistic of the posterior distribution as long as there are enough simulated samples from that distribution. The theory of MCMC techniques guarantees convergence, meaning that the samples generated will converge to a stationary distribution that is the target joint posterior that we are interested in (Gilks et al., 1996). In order to reach convergence, MCMC sampling needs to be applied for a large number of iterations. In addition, because the early iterations are biased, these samples need to be discarded. The discarded iterations are often referred to as the "burn-in" period (Yildirim, 2012).

Gibbs sampling represents an efficient inference MCMC algorithm (Gelfand \& Smith, 1990). The benefit of Gibbs sampling is that given a Bayesian network (e.g. like the one in Figure 6) it is simpler to sample from conditional distributions than to marginalize by integrating over a joint distribution. Gibbs sampling implies taking realizations of the distribution of each variable (in turn), conditional on the current values of the other variables. This sampling constitutes a Markov chain whose stationary distribution is the sought posterior joint distribution (Gelman et al., 2014).

It should be noted, however, that the proposed modeling approach is not the only alternative. For instance, the model specification could have followed the structure of GARCHtype models (i.e. Generalized AutoRegressive Conditional Heteroskedasticity (Engle, 1982)) or any other that presumably could be successful in the modeling of the relationship between lane-changing and average flow. In such cases, MCMC techniques can be applied on top of these models being advantageous on grounds of generality, accuracy and flexibility with respect to traditional estimation methods, like maximum likelihood, or the generalized method of moments (Vrontos et al., 2000). The number of different modelling alternatives could be large, and comparison between them would be interesting and left as an issue for further research.

\subsection{Model calibration for the B-23 case study}

Figure 7 summarizes step-by-step the proposed modeling approach, from the measurement of the input data to the model calibration. Note that the proposed Bayesian inference 
stochastic model was implemented in the OpenBUGS computing language, facilitating the coding of the model. This statistical modeling environment is especially suited to perform Bayesian inference using Gibbs sampling when fed with sample data and a candidate analytical model (Lunn et al., 2009). 10,000 iterations of the Gibbs sampler were run to ensure convergence of the algorithm, while the first 1,000 iterations were discarded as the burn-in period.

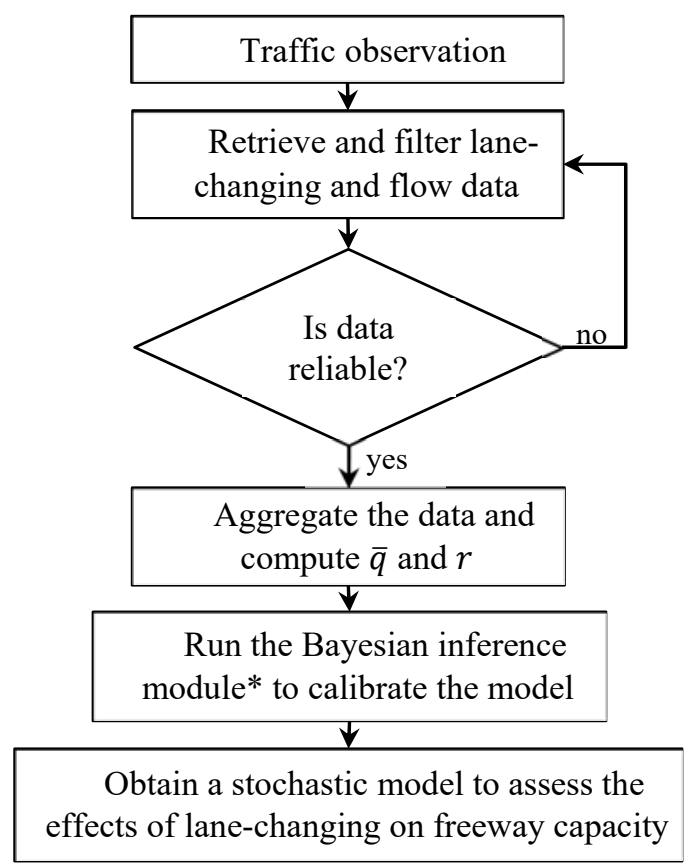

Figure 7. Step-by-step flowchart of the proposed methodology. Note: *Implemented in OpenBUGS.

Figure 8 shows the calibration results of the proposed stochastic model, when fed with data from detection zones 2304, 2305, 2306 and 2310, which are the ones with the best lane-changing data quality. The average flow per lane, $(\bar{q})$, is plotted with respect to the lane-changing normalized ratio $(r)$. Recall that $\bar{q}$ is computed by dividing the total sectional flow, $q$, by the number of lanes. In turn, $r$ represents the expected number of lane-changes for one vehicle traveling one $\mathrm{km}$ (see Equation 2). All data points are computed for aggregation periods of $\Delta t=3 \mathrm{~min}$. Data points are split in two separate sets representing freeflowing and congested traffic regimes, leading to two different calibrations of the model. They are represented, respectively, above and below the horizontal axis in Figure 8. In addition, Figure 8 shows the estimation for different percentiles of the distribution of $r$, and their variability. Note the narrow range between the $10^{\text {th }}$ and $90^{\text {th }}$ percentiles of these estimates (i.e. dotted lines in Figure 8) indicating an equivalently narrow distribution of the calibrated stochastic parameters. This proves the good fit of the model (see Figure 9 and Table 2). Wider distributions were obtained for other polynomial model specifications, indicative of a poorer fit (see Section 4.3).

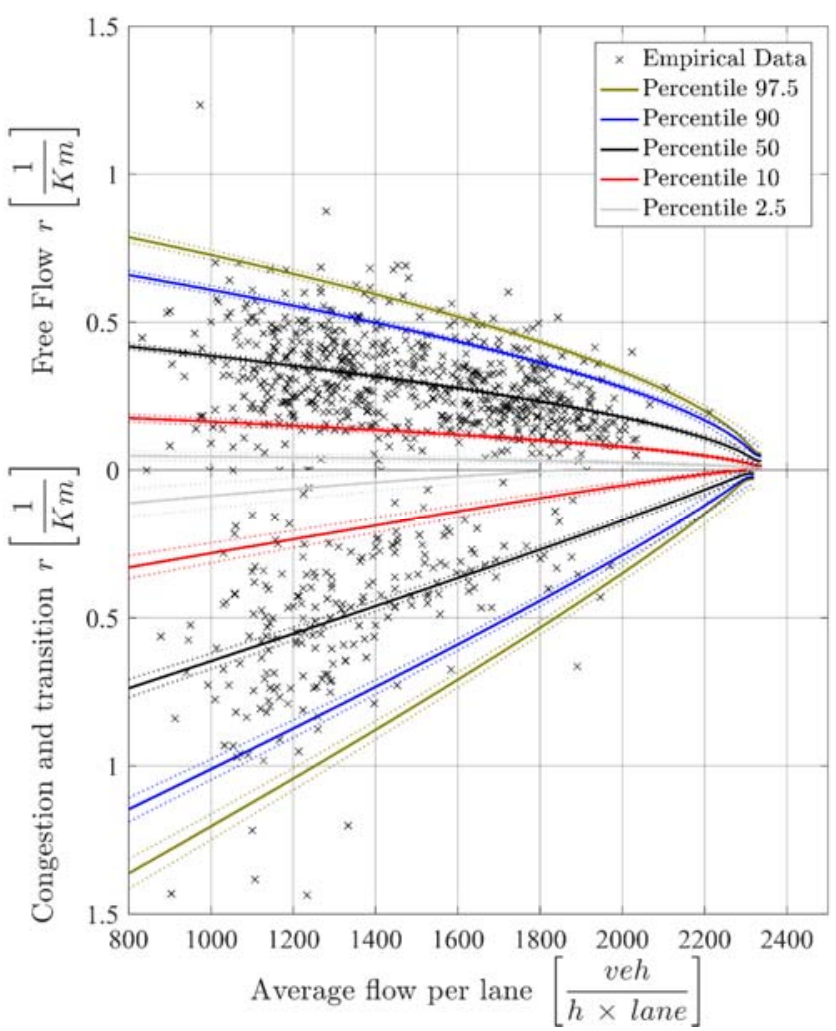

Figure 8. Lane-changing normalized ratio $(r)$ versus average flow per lane $(\bar{q})$. Note: 1$)$ All data points represent 3 min aggregation periods. 2) Data above the horizontal axis represent free-flowing traffic states. Data below the horizontal axis represent congested or transitional traffic states (i.e. at least one detector shows congestion during the whole or part of the $3 \mathrm{~min}$ period). 3) The two dotted lines beside percentile estimations show their 10\% (down) and $90 \%$ (up) confidence intervals.

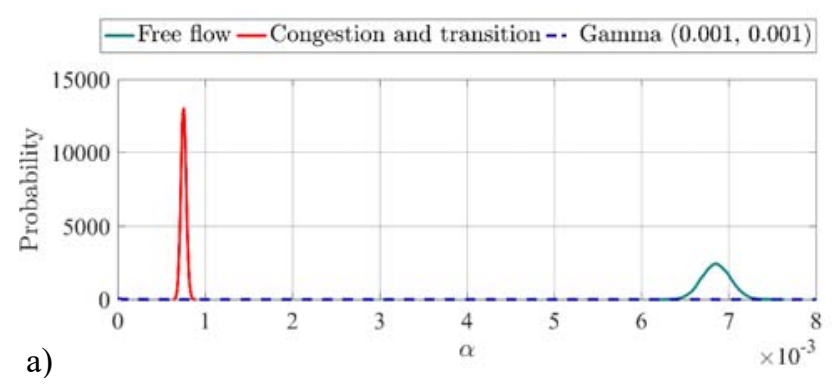



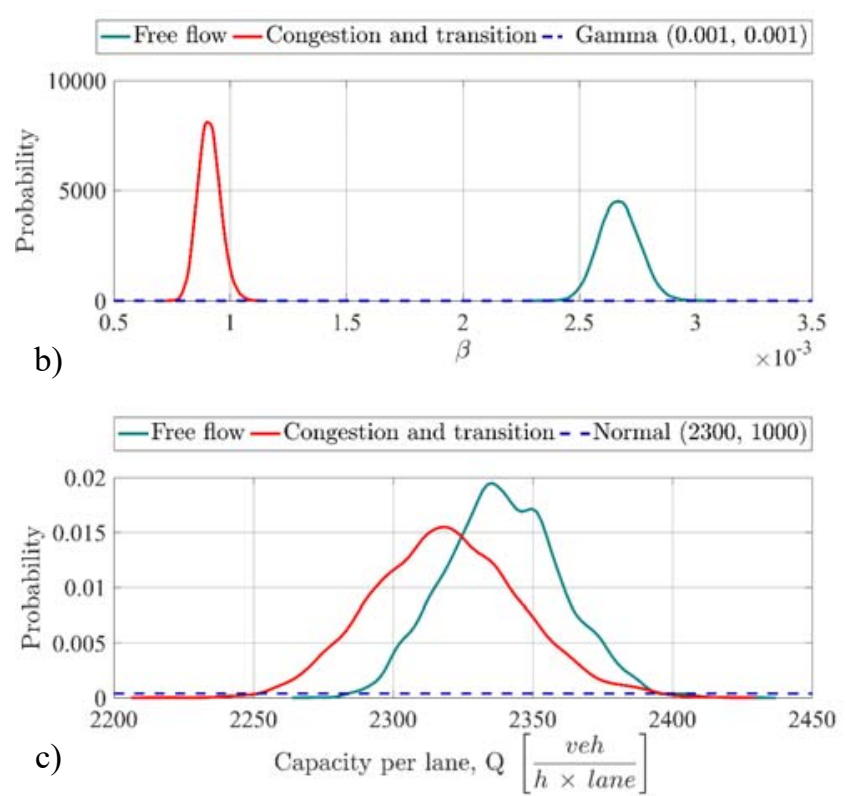

Figure 9. Probability density functions of the stochastic parameters $\alpha, \beta$ and $Q$ for the free-flowing and congested models. Note: The non-informative a-priori distributions are also presented (dashed blue series). This is aimed to show the robustness of the model to the input parameters.

Table 2

Calibrated parameters of the stochastic $r, \bar{q}$ models

\begin{tabular}{|ccc|}
\hline Parameter & Mean & $C V$ \\
\hline$\alpha_{f f}$ & $6.856 \cdot 10^{-3}$ & 0.02363 \\
$\gamma_{f f}$ & 0.56 & \\
$\beta_{f f}$ & $2.672 \cdot 10^{-3}$ & 0.03217 \\
$\delta_{f f}$ & 0.58 & \\
$Q_{f f}$ & $2339 \mathrm{veh} / \mathrm{h}$ & $8.937 \cdot 10^{-3}$ \\
\hline$\alpha_{\text {cong }}$ & $7.539 \cdot 10^{-4}$ & 0.04032 \\
$\gamma_{\text {cong }}$ & 0.94 & \\
$\beta_{\text {cong }}$ & $9.104 \cdot 10^{-4}$ & 0.05331 \\
$\delta_{\text {cong }}$ & 0.80 & \\
$Q_{\text {cong }}$ & $2319 \mathrm{veh} / \mathrm{h}$ & 0.01145 \\
\hline
\end{tabular}

*Subscripts " $f f$ " and "cong" refer to free-flowing and congested traffic. $\mathrm{CV}$ is the coefficient of variation (i.e. standard deviation to mean ratio).

\subsection{Sensitivity analysis}

Before further analyzing the results from the previous calibration of the model, a sensitivity analysis is presented in this section aiming to discuss the robustness and validity of the proposed model specification. To that end, different model specifications, in terms of the values of the polynomial rates (i.e. $\gamma$ and $\delta$ ), are tested and compared.
Comparison between Bayesian models is addressed using the deviance information criterion (DIC), which assesses the fit of the model to data taking into account the model complexity in terms of the effective number of parameters (Spiegelhalter et al., 2002). The deviance, $D(\mu, \sigma)$, is defined from the likelihood function as:

$$
D(\mu, \sigma)=-2 \log [p(\boldsymbol{R} \mid \mu, \sigma, \alpha, \beta, Q, \gamma, \delta)]
$$

And the effective number of parameters, $p_{D}$, is:

$$
p_{D}=\bar{D}-D(\bar{\mu}, \bar{\sigma})
$$

where $\bar{\mu}$ and $\bar{\sigma}$ are the expected values for the parameters of the normal distribution of $r$. Then, the DIC is calculated as in Equation 11.

$$
D I C=\bar{D}+p_{D}
$$

The concept is that models with smaller DIC should be preferred. Note that the models that receive the highest support from the data are those with the lowest values of $\bar{D}$. In addition, models are also penalized by $p_{D}$, compensating the fact that models with more parameters tend to fit data more easily (François \& Laval, 2011).

Figure 10 summarizes the different DIC's obtained for models with $\gamma$ and $\delta$ ranging from 0.5 to 1.1 . The sensitivity analysis in this range is enough, as it includes the models with minimum DIC, which are the ones selected. For freeflowing traffic regimes, a minimum DIC of -842.7 is obtained for $\gamma_{f f}=0.56$ and $\delta_{f f}=0.58$. For congested traffic states, the minimum DIC is -35.9 is obtained for $\gamma_{\text {cong }}=0.94$ and $\delta_{\text {cong }}=0.80$.

In addition, the $\mu[i]=1 / \bar{q}[i]$ model specification was also analyzed. This aims to analyze the fact that, with the previous definitions of $\bar{q}$ and $r$ and if the number of lanechanges $(n)$ is kept constant, the lane-changing normalized ratio $(r)$ would decrease with the flow, by definition, in the shape of $1 / \bar{q}$ instead of the polynomial decay (see Equations 1 and 2). The $1 / \bar{q}$ model specification was tested and resulted with larger DIC, showing the poorer performance of this model. In any case, it should be noted that a constant lanechanging count $(n)$ with increasing flows, actually represent a decrease in the unitary lane-changing activity. This means that, in spite of the definitions and variables used, the obtained insights are meaningful and representative of traffic behavior. 

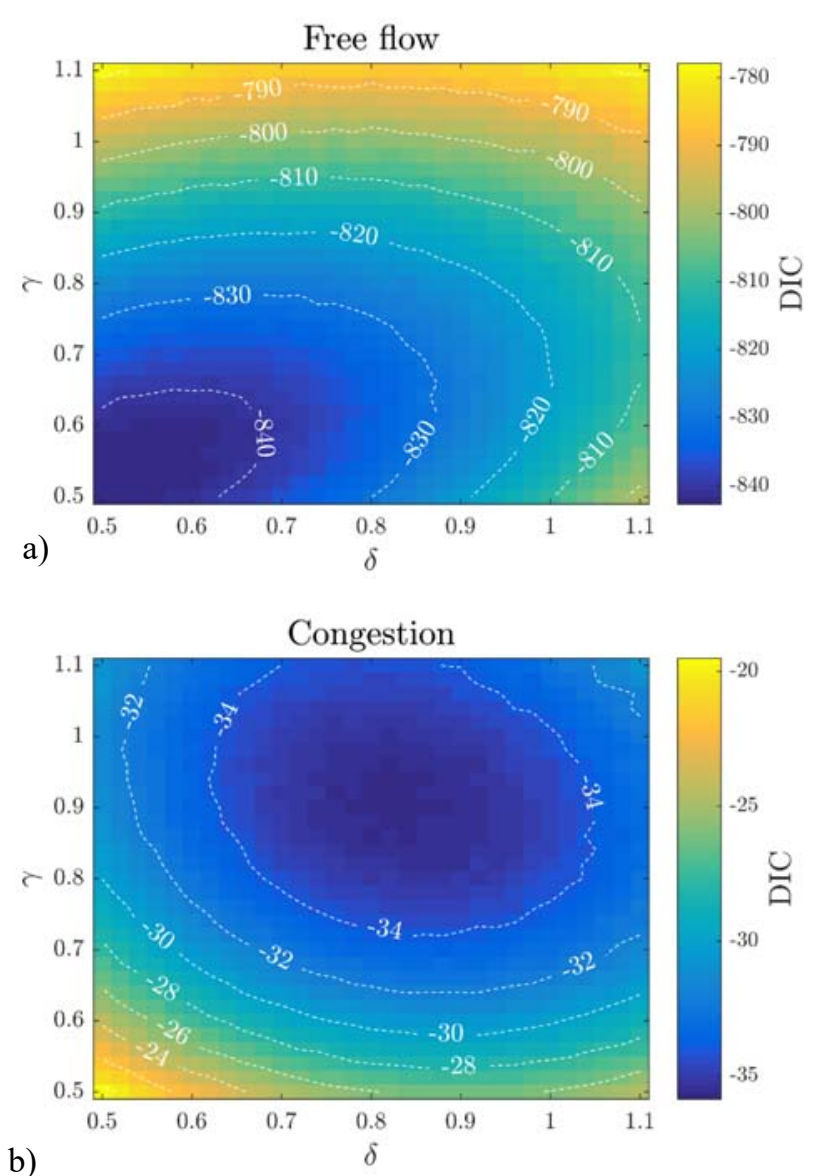

Figure 10. Sensitivity analysis of the model specification with respect to the polynomial rates $\gamma$ and $\delta$. Note: Deviance Information Criterion (DIC) is used as a goodness of fit measure. Lower DIC implies better fit.

\subsection{Discussion of obtained results}

Results in Figure 8 show that, for a given flow level, different $r$ values can be observed. Note that data from different freeway sections are included in Figure 8, and lane-changing is influenced by the presence of mergings and divergings with mandatory lane-changing and weaving sections. However, there exists an upper bound for the observed $r$, which decreases as the average flow per lane $(\bar{q})$ increases. This happens for both, free-flowing and congested traffic regimes. The possible explanation behind this relationship is that in free-flowing conditions, for larger flows, the opportunities to change lane became rarer, because traffic is fairly dense and therefore lanechanging becomes more difficult and, at the same time, less rewarding since not much can be gained by changing lanes. Still, lane-changing might be necessary in these dense traffic conditions due to the presence of a slow vehicle, random fluctuations in lane density, change in the lane geometry, etc. In such cases, this implies the increase of $r$, which might lead to the traffic breakdown, a reduction of the circulating flow, the increase of the variance of speed and density across lanes, and a further increase of the lanechanging. The observed upper bound in $r$ quantifies the threshold beyond which higher lane-changing activity would lead to a flow reduction. In other words, if $r$ increases beyond this threshold, the maximum flow that can be observed is smaller.

From the model results, it is particularly interesting the analysis of the parameter $Q$, the theoretical maximum capacity for the freeway section in ideal conditions, with no lane-changing activity (i.e. $s=r=0$; the model crossing with the horizontal axis in Figure 8). Before analyzing the obtained results, it should be understood that this scenario could be only observed by forbidding lane-changing activity in the target stretch. So far, this has not been possible, and the empirical validation of the model for $r$ tending to 0 remains as an issue for further research. In spite of this, Figure 9c shows the resulting probability density functions for $Q$ according to the stochastic model, in free-flowing and congested conditions. In free-flowing $Q$ is slightly larger, illustrating the capacity drop phenomenon in congested conditions. Furthermore, note that capacities larger than that currently observed at freeways could theoretically be achieved in the absence of lane-changing. Actually, almost the whole probability free-flowing distribution corresponds to values larger than those proposed in the HCM (2010) for the observed conditions in the test site (e.g. $2300 \mathrm{veh} / \mathrm{h} \cdot$ lane). This result unveils the potential of lane-changing restriction policies in improving traffic flow efficiency.

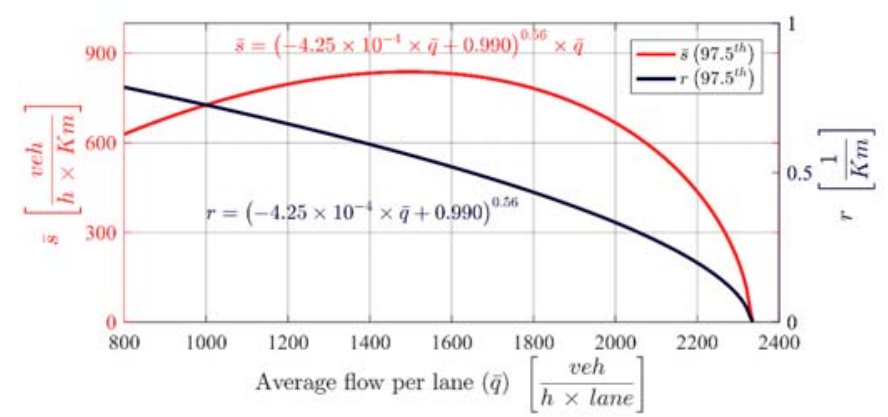

Figure 11. Maximum lane-changing per $\mathrm{km}$ and hour between any pair of lanes, $\bar{s}$, as a function of the average flow per lane, $\bar{q}$. Note: the $97.5^{\text {th }}$ percentile of the distribution of $r$ is considered as the upper lane-changing bound. $\bar{s}$ is obtained by multiplying $r$ 's $97.5^{\text {th }}$ percentile times $\bar{q}$.

To that end, Figure 11 is proposed as a tool to support traffic management strategies involving lane-changing 
restrictions. This figure shows the maximum number of lane-changes that can take place if a certain flow value is to be sustained. Note that the percentile $97.5^{\text {th }}$ of the freeflowing $r$ distribution was selected to represent the upper bound for lane-changing. The free-flowing distribution is chosen because any traffic management strategy must aim to keep or recover free-flowing traffic conditions. Also note that for most of the flows, the maximum acceptable lane-changing activity is lower or equal in free-flowing than in congested conditions. The $97.5^{\text {th }}$ percentile selection is rather arbitrary, and any other percentile could be used, yielding to the same conceptual results but with different values, as discussed later. In Figure 11, the normalized lane-changing ratio $(r)$ is shown together with the per lane lane-changing flow $(\bar{s})$ [lanechanges $/ \mathrm{km} \cdot \mathrm{h} \cdot$ lane]. $\bar{s}$ is simply obtained by multiplying the $r^{\prime}$ s $97.5^{\text {th }}$ percentile, times the corresponding average flow, $\bar{q}$. This results in a lane-changing model for the $97.5^{\text {th }}$ percentile of $\bar{s}$. From this model it can be seen that for small flows, an increase of $\bar{q}$ results in a decrease of $r$ but an increase of $\bar{s}$. This means that, even if each vehicle is less likely to change lanes (lower $r$ ), this is compensated by the increasing number of vehicles in the freeway, so that the $\bar{s}$ can be higher. Results for flows lower than 800 $\mathrm{veh} /(\mathrm{h} \cdot \mathrm{lane})$ should be taken with caution and are not represented in Figure 11 as few data were collected in this region. Nevertheless, Figure 11 is more interesting for flows higher than that of the tipping point (in this case a maximum of $\bar{s}=837 \quad$ [lane-changes $/ \mathrm{km} \cdot \mathrm{h} \cdot$ lane], corresponding to $r=0.56$ [lane-changes $/ \mathrm{veh} \cdot \mathrm{km}$ ] when the circulating flow is $1492[\mathrm{veh} / \mathrm{h} \cdot$ lane]) from where the increase of flow does not longer compensate the reduction of $r$. In this region, Figure 11 can be interpreted as the maximum lane-changing activity that can happen in order to maintain a throughput level. If $\bar{s}$ (or $r$ ) goes above this threshold, the flow will inevitably decrease.

Given the previous interpretation, the model could be useful for lane-changing control applications, as it determines the maximum acceptable number of lanechanges in order to ensure a desired throughput of the freeway section (see Figure 12). Note that the percentile chosen for the upper bound of $r$ would represent a tuning parameter of the control algorithm. In the previous example, the $97.5^{\text {th }}$ percentile was chosen. This responds to a compromise between the reliability and the severity of the control strategy. Smaller percentiles would increase the reliability in the achievement of the desired flows at the cost of a more restrictive policy, while larger ones would behave in the opposite way: being less reliable but also less restrictive. In fact, the model provides the $r$ distribution for any $\bar{q}$. So, in a particular application, any desired probability level could be chosen in the tuning of the control algorithm. This concept is similar to the Sustained Flow Index (SFI) that addresses the trade-off between flow and reliability (Shojaat et al., 2016).

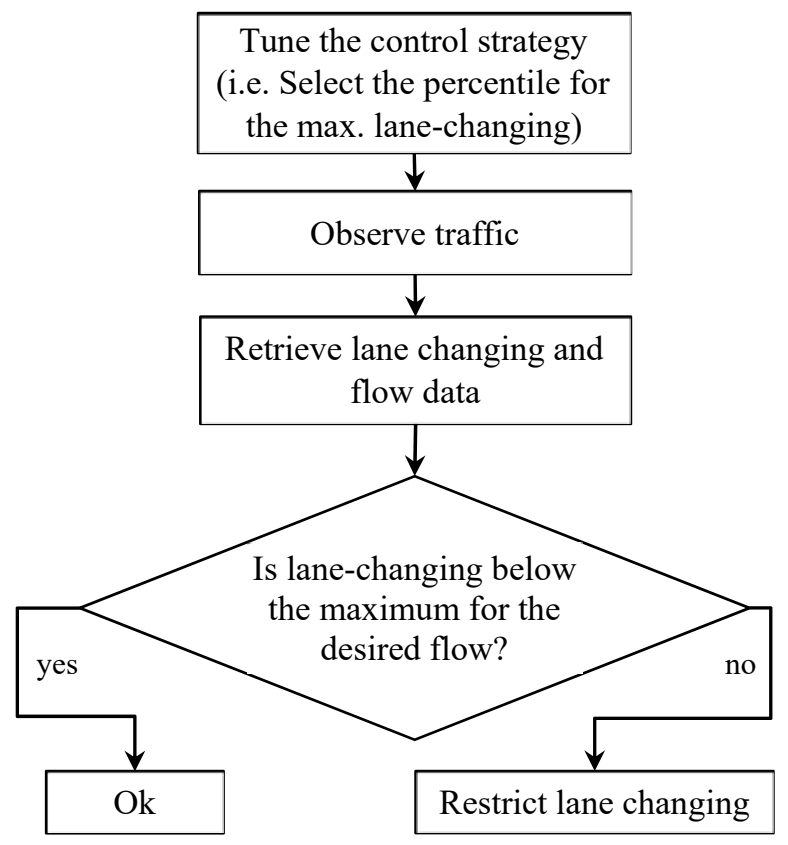

Figure 12. Step-by-step flowchart of the proposed lanechanging control application.

In practice, with the current technology installed in most freeways, everything different from all-or-nothing control (i.e. allow or prohibit) lane-changing might be difficult to implement. In light of this limitation, one strategy could be to prohibit lane-changing when traffic flow reaches a level where small lane-changing ratios could lead to traffic breakdown. Actually, this may be enough in most of the realworld situations, as the objective would be to achieve the maximum possible capacity, $Q$. An alternative to explore could be the management of vehicles' desired speeds. This control strategy might modify vehicles' speed distribution and consequently impact lane-changing rates. This could be attempted by using dynamic speed limit (DSL) strategies to achieve more uniform travelling speeds and reduce lanechanging rates. In such case, strict enforcement all along the freeway section (e.g. travel time control) would be needed. Otherwise, DSL may lead to the opposite behavior (i.e. increase of lane-changing rates) (Soriguera et al., 2017). In the next future, with the advent of V2I communications, Advanced Driver-Assistance Systems (ADAS) and vehicle automation, lane-changing control could be feasible by 
efficiently distributing lane-changing maneuvers amongst vehicles in time and space so that the target lane-changing ratio $(r)$ could be precisely matched.

\section{CONCLUSIONS}

Lane-changing activity is one of the major disruptors in freeway traffic and a key contributor to traffic instabilities and capacity drop. The research presented in this paper supports this disruptor role of lane-changing in freeways. It has been observed, for multiple locations and days, that lane-changing activity peaks during congestion. Even more, the largest concentration of lane-changes is generally located around shockwaves, at the transition between traffic states. Although no direct evidence has been found confirming that lane-changing activity triggers congestion episodes, a clear relationship between the maximum lane change activity and the maximum average flow per lane has been observed. The lower the maximum lane-changing rate, the larger the maximum flow per lane that can be sustained.

In order to model these qualitative observations, a Bayesian inference stochastic approach is used. This enables to define analytically the relationship between the freeway capacity reduction and the lane-changing activity. Specifically, the normalized lane-changing rate $(r)$ is defined by a heteroscedastic normal distribution with mean and standard deviation decreasing with the average flow per lane $(\bar{q})$. This model is useful to determine the maximum lane-changing rate that can be supported in order to ensure a given freeway throughput.

These findings quantify the prevailing theories in the literature arguing that lane-changes are an important traffic disruptor that can trigger congestion. In consequence, largest flows can only be achieved with very low lane-changing rates, as any disruption is enough to breakdown traffic in this highly synchronized flow. Therefore, the theoretical maximum flow (i.e. the capacity) would be achieved in the no lane-changing scenario. For this ideal scenario, the proposed stochastic model is used to derive the probability density function for capacity. The obtained capacity values are somehow larger than the ones empirically observed and proposed in manuals. This confirms the potential of lane-changing control strategies during peak periods in order to improve traffic efficiency. Otherwise, lane-changing can potentially make traffic unstable leading to a capacity reduction (i.e. a capacity drop). Obviously, such control strategies must deal with the existence of mandatory lanechanges near diverging segments.

\section{ACKNOWLEDGEMENTS}

Authors acknowledge the collaboration of Mr. Adrià Torres in the counting of lane-changes from video recordings. Also, the comments of Mrs. Margarita Martínez to preliminary versions of the paper have had a positive impact in this final version. The database construction would not have been possible without the collaboration of the Catalan Traffic Administration (Servei Català de Trànsit) and the staff at the Traffic Management Center. The guidance of Prof. Enrique Castillo in the usage of the OpenBUGS software is also gratefully acknowledged. This research has been partially funded by the Spanish Ministry of Economy and Competitiveness (Ministerio de Economía y Competitividad, Gobierno de España), grant number TRA2016-79019-R/COOP.

\section{REFERENCES}

Ahn, S., \& Cassidy, M. J. (2007). Freeway traffic oscillations and vehicle lane-change maneuvers. 17th International Symposium Of Transportation and Traffic Theory.

Banks, J. H. (1991). Two-capacity phenomenon at freeway bottlenecks: A basis for ramp metering. Transportation Research Record, 1320, 83-90.

Brilon, W., Geistefeldt, J., \& Regler, M. (2005). Reliability of freeway traffic flow: a stochastic concept of capacity. Proceedings of the 16th International Symposium on Transportation and Traffic Theory, 125143.

Cassidy, M. J., \& Bertini, R. L. (1999). Some traffic features at freeway bottlenecks. Transportation Research Part B: Methodological, 33(1), 25-42.

Cassidy, M. J., Jang, K., \& Daganzo, C. F. (2010). The smoothing effect of carpool lanes on freeway bottlenecks. Transportation Research Part A: Policy and Practice, 44(2), 65-75.

Cassidy, M. J., \& Rudjanakanoknad, J. (2005). Increasing the capacity of an isolated merge by metering its on-ramp. Transportation Research Part B: Methodological, 39(10), 896-913.

Cassidy, M. J., \& Windover, J. R. (1995). Methodology for assessing dynamics of freeway traffic flow. Transportation Research Record, 1484, 73-79.

Castillo, E., Calviño, A., Grande, Z., Sánchez-Cambronero, S., Gallego, I., Rivas, A., \& Menéndez, J. M. (2016a). A Markovian-Bayesian network for risk analysis of high speed and conventional railway lines integrating human errors. Computer-Aided Civil and Infrastructure Engineering, 31(3), 193-218.

Castillo, E., Grande, Z., \& Calviño, A. (2016b). Bayesian 
networks-based probabilistic safety analysis for railway lines. Computer-Aided Civil and Infrastructure Engineering, 31(9), 681-700.

Castillo, E., Grande, Z., Mora, E., Lo, H. K., \& Xu, X. (2017a). Complexity reduction and sensitivity analysis in road probabilistic safety assessment bayesian network models. Computer-Aided Civil and Infrastructure Engineering, 32(7), 546-561.

Castillo, E., Grande, Z., Mora, E., Xu, X., \& Lo, H. K. (2017b). Proactive, backward analysis and learning in road probabilistic Bayesian network models. ComputerAided Civil and Infrastructure Engineering, 32(10), 820-835.

Chen, D., Ahn, S., Laval, J., Zheng, Z. (2014). On the periodicity of traffic oscillations and capacity drop: the role of driver characteristics. Transportation Research: Part B: Methodological, 59, 117-136.

Chen, D., \& Ahn, S. (2018). Capacity-drop at extended bottlenecks: Merge, diverge, and weave. Transportation Research Part B: Methodological, 108, 1-20.

Chung, K., Rudjanakanoknad, J., \& Cassidy, M. J. (2007). Relation between traffic density and capacity drop at three freeway bottlenecks. Transportation Research Part B: Methodological, 41(1), 82-95.

Coifman, B., \& Li, L. (2017). A critical evaluation of the Next Generation Simulation (NGSIM) vehicle trajectory dataset. Transportation Research Part B: Methodological, 105, 362-377.

Coifman, B., Mishalani, R., Wang, C., Krishnamurthy, S., \& Harris, A. D. (2006). Impact of lane-change maneuvers on congested freeway segment delays: Pilot study. Transportation Research Record, 1965, 152159.

Deublein, M., Matthias, S., Adey, B. T., \& García de Soto, B. (2015). A Bayesian network model to predict accidents on Swiss highways. Infrastructure Asset Management, 2(4), 145-158.

Elefteriadou, L., Roess, R. P., \& McShane, W. R. (1995). Probabilistic nature of breakdown at freeway merge junctions. Transportation Research Record, 1484, 8089.

Elefteriadou, L., Roess, R.P., \& McShane, W.R. (2005). Probabilistic nature of breakdown at freeway merge junctions. Transportation Research Record, 1484, 8089.

Engle, R. F. (1982). Autoregressive conditional heteroscedasticity with estimates of the variance of United Kingdom inflation. Econometrica, 50(4), 9871007.

Federal Highway Administration. (2006). Interstate 80
Freeway Dataset Factsheet. Fhwa-Hrt-06-137. Retrieved from:

http://www.fhwa.dot.gov/publications/research/operation s/06137/

Federal Highway Administration. (2015). Next Generation Simulation (NGSIM). Retrieved March 3, 2016, from: http://ops.fhwa.dot.gov/trafficanalysistools/ngsim.htm.

François, O., \& Laval, G. (2011). Deviance information criteria for model selection in approximate Bayesian computation. Statistical Applications in Genetics and Molecular Biology, 10(1), 1-25.

Gilks, W. R., Richardson, S., \& Spiegelhalter, D. J. (1996). Markov Chain Monte Carlo in practice. London: Chapman and Hall.

Gelfand A. E. \& Smith A. F. M. (1990). Sampling-based approaches to calculating marginal densities. Journal of the American Statistical Association, 85, 398-409.

Geistefeldt, J., \& Brilon, W. (2009). A comparative assessment of stochastic capacity estimation methods. In: Lam W., Wong S., Lo H. (eds) Transportation and Traffic Theory 2009: Golden Jubilee. Springer, Boston, MA.

Gelman, A., Carlin, J. B., Stern, H. S., Dunson, D. B., Vehtari, A. \& Rubin, D. B. (2014). Bayesian data analysis. CRC Texts in Statistical Science. Chapman \& Hall.

Ghofrani, F., Pathak, A., Mohammadi, R., Aref, A., and He, Q. (2019). Predicting rail defect frequency: An integrated approach using fatigue modeling and data analytics. Computer-Aided Civil and Infrastructure Engineering, 115.

Goñi-Ros, B., Knoop, V.L., Takahashi, T., Sakata, I., van Arem, B., Hoogendoorn, S.P. (2016). Optimization of traffic flow at freeway sags by controlling the acceleration of vehicles equipped with in-car systems. Transportation Research: Part C: Emerging Technologies, 71, 1-18.

Hall, F. L., \& Agyemang-Duah, K. (1991). Freeway capacity drop and the definition of capacity. Transportation Research Record, 1320, 91-98.

Hashemi, H., \& Abdelghany, K. (2018). End-to-end deep learning methodology for real-time traffic network management. Computer-Aided Civil and Infrastructure Engineering, 33(10), 849-863.

Hatakenaka, H., Hirasawa, T., Yamada, K., Yamada, H., Katayama, Y., \& Maeda, M. (2004). Development of AHS for Traffic Congestion in SAG Sections. $13^{\text {th }}$ World Congress on ITS.

Highway Capacity Manual. (2010). Transportation Research Board, National Research Council, Washington, DC.

Hossain, M., \& Muromachi, Y. (2012). A Bayesian network based framework for real-time crash prediction on the basic freeway segments of urban expressways. Accident Analysis \& Prevention, 45, 373-381. 
Huang, Y., \& Beck, J. L. (2018). Full Gibbs sampling procedure for Bayesian system identification incorporating sparse Bayesian learning with automatic relevance determination. Computer-Aided Civil and Infrastructure Engineering.

Ibrahim, A. T., \& Hall, F. L. (1994). Effect of adverse weather conditions on speed-flow occupancy relationships. Transportation Research Record, 1457, 184-191.

Jia, A., Zhou, X., Li, M., Rouphail, N. M., \& Williams, B. M. (2011). Incorporating stochastic road capacity into day-to-day traffic simulation and traveler learning framework: Model development and case study. Transportation Research Record, 2254(1), 112-121.

Jin, W. L. (2010). A kinematic wave theory of lanechanging traffic flow. Transportation Research Part B: Methodological, 44(8-9), 1001-1021.

Jin, C. J., Knoop, V. L., Li, D., Meng, L. Y., \& Wang, H. (2019). Discretionary lane-changing behavior: empirical validation for one realistic rule-based model. Transportmetrica A: Transport Science, 15(2), 244262.

Knoop, V. , Hoogendoorn, S. , Adams, K. (2009). Capacity reductions at incidents sites on motorways. European Journal of Transportation and Infrastructures Research, 9, 363-379.

Kosgodagan, A., Morales-Napoles, O., Yeung, T., Courage, W., Maljaars, J., and Castanier, B. (2017). A Two-dimension dynamic Bayesian network for largescale degradation modelling with an application to a bridges network. Computer-Aided Civil and Infrastructure Engineering, 32(8), 641-656.

Lamm, R., Choueiri, E. M., \& Mailaender, T. (1987). Comparison of operating speed on dry and wet pavement of two lane rural highways. Transportation Research Record, 1280, 199-207.

Laval, J. A., \& Daganzo, C. F. (2006). Lane-changing in traffic streams. Transportation Research Part B: Methodological, 40(3), 251-264.

Leclercq, L., Laval, J. A., \& Chiabaut, N. (2011). Capacity drops at merges: An endogenous model. ProcediaSocial and Behavioral Sciences, 17, 12-26.

Liu, Q., Wang, B., \& Zhu, Y. (2018). Short-term traffic speed forecasting based on attention convolutional neural network for arterials. Computer-Aided Civil and Infrastructure Engineering, 33(11), 999-1016.

Lorenz, M., \& Elefteriadou, L. (2000). A probabilistic approach to defining freeway capacity and breakdown. TRB-Circular E-C018, Transportation Research Board, 84-95.

Lunn, D., Spiegelhalter, D., Thomas, A. \& Best, N. (2009).
The BUGS project: Evolution, critique and future directions. Statistics in Medicine, 28, 3049-3067.

Lv. Y., Duan, Y., Kang, W., \& Wang, F. Y. (2015). Traffic flow prediction with big data: A deep learning approach. IEEE Trans. Intelligent Transportation Systems, 16(2), 865-873.

Marczak, F., Daamen, W., \& Buisson, C. (2014). Empirical analysis of lane changing behaviour at a freeway weaving section. Transport Research Arena (TRA) 5th Conference: Transport Solutions from Research to Deployment, Institut Francais des Sciences et Technologies des Transports, de l'Aménagement et des Réseaux. IFSTTAR.

Marczak, F., Leclercq, L. \& Buisson, C. (2015). A Macroscopic model for freeway weaving sections. Computer-Aided Civil and Infrastructure Engineering, 30(6), 464-477.

Mauch, M., \& Cassidy, M. J. (2002). Freeway traffic oscillations: observations and predictions. Transportation and Traffic Theory in the 21st Century: Proceedings of the 15th International Symposium on Transportation and Traffic Theory, Adelaide, Australia, 16-18 July 2002, 653673.

Menendez, M., \& Daganzo, C. F. (2007). Effects of HOV lanes on freeway bottlenecks. Transportation Research Part B: Methodological, 41(8), 809-822.

Mihaylova, L., Boel, R., \& Hegyi, A. (2007). Freeway traffic estimation within particle filtering framework. Automatica, 43(2), 290-300.

Minderhoud, M. M., Botma, H. B., \& Bovy, P. H. L. (1997). Assessment of roadway capacity estimation methods. Transportation Research Record, 1572, 59-67.

Montanino, M., \& Punzo, V. (2015). Trajectory data reconstruction and simulation-based validation against macroscopic traffic patterns. Transportation Research Part B: Methodological, 80, 82-106.

Muñoz, J.C., \& Daganzo, C.F. (2002). The bottleneck mechanism of a freeway diverge. Transportation Research Part A: Policy and Practice, 36(6), 483-505.

Oh, S., \& Yeo, H. (2012). Estimation of capacity drop in highway merging sections. Transportation Research Record, 2286(1), 111-121.

Oh, S., Yeo, H. (2015). Impact of stop-and-go waves and lane changes on discharge rate in recovery flow. Transportation Research: Part B Methodological, 77, 88102.

Okamura, H., Watanabe, S., \& Watanabe, T. (2000). An empirical study on the capacity of bottlenecks on the basic suburban expressway sections in Japan. Proceedings of the 4th International Symposium on Highway Capacity, 12, 120-129.

de Oña, J., Mujalli, R. O., \& Calvo, F. J. (2011). Analysis of 
traffic accident injury severity on Spanish rural highways using Bayesian networks. Accident Analysis and Prevention, 43(1), 402-411.

Ozbay, K., \& Ozguven, E. E. (2007). A comparative methodology for estimating the capacity of a freeway section. IEEE Intelligent Transportation Systems Conference, 1034-1039. Seattle, WA.

Patire, A. D., \& Cassidy, M. J. (2011). Lane changing patterns of bane and benefit: Observations of an uphill expressway. Transportation Research Part B: Methodological, 45(4), 656-666.

Persaud, B., Yagar, S., \& Brownlee, R. (1998). Exploration of the breakdown phenomenon in freeway traffic. Transportation Research Record, 1634, 64-69.

Polus, A. \& Pollatschek, M. A. (2001). Stochastic nature of freeway capacity and its estimation. Canadian Journal of Civil Engineering, 29(6), 842-852.

Punzo, V., Borzacchiello, M. T., \& Ciuffo, B. (2011). On the assessment of vehicle trajectory data accuracy and application to the Next Generation SIMulation (NGSIM) program data. Transportation Research Part C: Emerging Technologies, 19(6), 1243-1262.

Sala, M., Soriguera, F., Huillca, K., \& Vilaplana, V. (2019). Measuring traffic lane-changing by converting video into space-time still images. Computer-Aided Civil and Infrastructure Engineering, 34(6), 488-505.

Shojaat, S., Geistefeldt, J., Parr, S. A., Wilmot, C. G., \& Wolshon, B. (2016). Sustained flow index: Stochastic measure of freeway performance. Transportation Research Record, 2554, 158-165.

Smith, B. L., Byrne, K. G., Copperman, R. B., Hennessy, S. M., \& Goodall, N. J. (2004). An investigation into the impact of rainfall on freeway traffic flow. In $83 \mathrm{rd}$ Annual Meeting of the Transportation Research Board, Washington DC.

Soriguera, F., Martínez, I., Sala, M., \& Menéndez, M. (2017). Effects of low speed limits on freeway traffic flow. Transportation Research Part C: Emerging Technologies, 77, 257-274.

Soriguera, F., \& Sala, M. (2014). Experimenting with dynamic speed limits on freeways. Procedia Social and Behavioral Sciences, 160, 35-44.

Spiegelhalter D. J., Best, N. G., Carlin, B. P., \& van der Linde, A. (2002) Bayesian measures of model complexity and fit (with discussion). Journal of the Royal Statistical Society Series B, 64(4), 583-639.

Srivastava, A., \& Geroliminis, N. (2013). Empirical observations of capacity drop in freeway merges with ramp control and integration in a first-order model. Transportation Research Part C: Emerging Technologies, 30, 161-177.
Sun, D. J., \& Elefteriadou, L. (2012). Lane-changing behavior on urban streets: An "in-vehicle" field experiment-based study. Computer-Aided Civil and Infrastructure Engineering, 27(7), 525-542.

Sun, D. J., \& Kondyli, A. (2010). Modeling vehicle interactions during lane-changing behavior on arterial streets. Computer-Aided Civil and Infrastructure Engineering, 25(8), 557-571.

Sun, S., Zhang, C., \& Yu, G. (2006). A Bayesian network approach to traffic flow forecasting. IEEE Transactions on Intelligent Transportation Systems, 7(1), 124-133.

Tajalli, M., \& Hajbabaie, A. (2018). Dynamic speed harmonization in connected urban street networks. Computer-Aided Civil and Infrastructure Engineering, 33(6), 510-523.

Vrontos, I. D., Dellaportas, P., \& Politis, D. N. (2000). Full Bayesian inference for GARCH and EGARCH models. Journal of Business \& Economic Statistics, 18(2), 187198.

Wang, C., \& Coifman, B. (2008). The effect of lane-change maneuvers on a simplified car-following theory. IEEE Transactions on Intelligent Transportation Systems, 9(3), 523-535.

Yao, B., Chen, C., Cao, Q., Jin, L., Zhang, M., Zhu, H., \& Yu, B. (2017). Short-term traffic speed prediction for an urban corridor. Computer-Aided Civil and Infrastructure Engineering, 32(2), 154-169.

Yeon, J., Hernandez, S., \& Elefteriadou, L. (2009). Differences in freeway capacity by day of the week, time of day, and segment type. Journal of Transportation Engineering, 135(7), 416-426.

Yildirim, I. (2012). Bayesian inference: Gibbs sampling. Technical Note, University of Rochester.

Yin, H., Wong, S., Xu, J., \& Wong, C. K. (2002). Urban traffic flow prediction using a fuzzy-neural approach. Transportation Research Part C: Emerging Technologies, 10(2), 85-98.

Yuan, K., Laval, J., Knoop, V. L., Jiang, R., \& Hoogendoorn, S. P. (2019). A geometric Brownian motion car-following model: towards a better understanding of capacity drop, Transportmetrica B: Transport Dynamics, 7:1, 915-927.

Yuan, K., Knoop, V. L., \& Hoogendoorn, S. P. (2015). Capacity drop relationship between speed in congestion and the queue discharge rate. Transportation Research Record, 2491, 72-80.

Yuen, K.X. and Huang, K. (2018). Identifiability-enhanced Bayesian Frequency-domain Substructure Identification. Computer-Aided Civil and Infrastructure Engineering, 33(9), 800-812. 